\title{
LOS FONEMAS SEGMENTALES DEL PROTOLENCA: RECONSTRUCCION COMPARATIVA
}

Gilda Rosa Arguedas Cortés

\begin{abstract}
The purpose of this article is to reconstruct the segmental phonemes of Proto-Lencan using the material available on Honduran and Salvadoran Lenca (the two languages that comprise the Lencan family) as a basis for comparison.

The study consist of: a) an introduction to the languages, a review of the current state of diachronic study of the Lencan family, the theoretical framework and the data sources; b) the phonemes of the languages compared; c) a reconstruction of the Proto-Lencan phonemic system and its reflex an descendent languages; d) a list of cognates.
\end{abstract}

\section{INTRODUCCION}

\subsection{Las lenguas lencas. Fuentes para su estudio}

La familia lenca (Campbell, 1979: 939 está integrada por dos lenguas: el lenca salvadoreño y el lenca hondureño. Swadesh (1967:98) calculó glotocronológicamente una separación de dos mil años entre ambas.

Los datos del lenca salvadoreño, que con seguridad está extinto (Campbell, 1976a: 73), están contenidos en Lehmann (1920: 700-719) y Campbell (1976a: 73-78) y fueron analizados por del Río Urrutia (1985). Concretamente, se trata de los siguientes materiales:

a) Datos recogidos personalmente por Lehmann en 1909 (1920: 700-719). Incluyen una lista de alrededor de 600 términos, conjugaciones de formas verbales y algunas frases. Se trata del corpus más completo y sus transcripciones son relativamente confiables gracias a su conocimiento de la fonética.

b) Datos recogidos por Atilio Peccorini. (Lehmann, 1920: 693-699) provenientes de un manuscrito del que Lehmann obtuvo una copia en Génova en 1911. Están integrados por una lista de unos 300 términos, conjugaciones parciales de 10 verbos y 45 frases. La transcripción es defectuosa. c) Datos recogidos por Karl Sapper en 1885 (Lehmann, 1920: 692) provenientes de un manuscrito del que Lehmann obtuvo una copia en el Salvador en 1909. Se trata de una lista de 20 términos. La transcripción es defectuosa.

ch) Datos de un manuscrito conservado por el alcalde de Chilanga (pueblo salvadoreño del cual proceden todos los testimonios que han quedado de la lengua) del cual Lehmann obtuvo copia en 1909. Están constituidos por una lista de 16 términos. La transcripción es defectuosa.

d) Datos recogidos por Lyle Campbell (1976a) al último hablante de la lengua en 1970 antes de su muerte. Están integrados por una lista de 92 términos y 25 frases. La transcripción, aunque fonéticamente menos detallada que la de Lehmann, es la más consistente.

El lenca hondureño probablemente esté extinto también (Campbell et alii, 1978: 331), aunque no existe total seguridad sobre esto. La lengua está más pobremente atestiguada que el lenca salvadoreño y la transcripción de los datos, cuyas fuentes se citan a continuación, es defectuosa con la única excepción de los de fecha más reciente:

a) Datos recogidos por Ephraim G. Squier (1858: 253-255) en las localidades de Guaji- 
quiro, Opatoro y Similatón (actualmente Cabañas) del departamento de La Paz, y Yamalanguira del departamento de Intibucá. Se trata de listas de, respectivamente, 93, 78, 55 y 37 términos. Lehmann los incluyó en su obra (1920: 669-670).

b) Datos recogidos por Alphonse L. Pinart (1897) en Guajiquiro. Incluyen 165 rubros entre palabras y frases breves. Lehmann los incluyó en su obra (1920: 671-686).

c) Datos de Guajiquiro recogidos por Alberto Membreño (1897: 247-250). Lehmann los incluyó en su obra (1920: 671-688). Abarcan dos listas: una de 402 palabras y frases cortas y otra de 63 oraciones.

ch) Datos de Similatón recogidos por Alberto Membreño (1897: 255-260). Lehmann los incluyó en su obra (1920: 692). Abarcan una lista de 264 términos, formas de la conjugación de un verbo en varios tiempos y 15 oraciones.

d) Datos recogidos por Anne Chapman en 1965 en Intibucá (Campbell et alii, 1978). Se trata de una lista de 38 rubros entre palabras y frases breves.

\subsection{Localización geográfica de los pueblos len- cas y características culturales}

De acuerdo con Chapman (1978), al momento de la llegada de los españoles, los lencas ocupaban un territorio que abarcaba parte de las actuales repúblicas de Honduras y de El Salvador y que estaba organizado en las siguientes regiones:

1. Care: departamento de La Paz, norte del departamento de Intibucá, norte del departamento de Lempira y sur del de Santa Bárbara en Honduras. De esta zona proceden los datos del lenca de Honduras (Similatón, Guajiquiro y Opatoro), como se señaló, están en La Paz; Yamalanguira e Intibucá, en Intibucá).

2. Cerquín: centro y sur del departamento de Lempira y sur del de Intibucá en Honduras.

3. Lenca: departamento de Comayagua, este del departamento de La Paz, centro y sur del departamento de Francisco Morazán, departamento del Valle y este del departamento de Choluteca en Honduras.

4. Potón: zona de El Salvador situada al oeste del río Lempa. En ella se encuentra el poblado de Chilanga del que, como se señaló, provienen todos los datos del lenca salvadoreño.
Culturalmente, los lencas presentaban rasgos predominantemente mesoamericanos (Chapman: 1978:37): sociedad estratificada (con jerarquía de nobles, vasallos y esclavos), agricultura en gran escala y concentraciones de población en poblados relativamente grandes.

\subsection{Posición de la familia lenca en las clasifica- ciones de las lenguas americanas}

Si bien hasta el momento no se han publicado estudios diacrónicos sistemáticos de las lenguas lencas, en las diversas clasificaciones de las lenguas americanas se les han asignado diversas relaciones con otras familias lingüísticas. A continuación se ofrece una síntesis de la forma en que la familia ha sido tratada desde este punto de vista.

Thomas (1902: 209) incluye la familia lenca en su lista de familias lingüísticas de Méjico y Centroamérica sin vincularla con ninguna otra.

Schmidt (1926: 206-208) incluye las lenguas lencas en un grupo misquito-xinca dividido en dos subgrupos: el paya-xinca (dividido en paya-jicaque, lenca y xinca) y el misquito-matagalpa.

Mason (1939: 238) incluye a la familia lenca entre los grupos no clasificados de América Central, si bien señala que se han postulado relaciones a nivel de filo tanto con el mixe-zoque (penuncio) como con el macrochibcha. En 1940 (p. 74), mantiene la misma clasificación, pero añade que se han postulado relaciones también con el filo hoca. En 1943 (p. 241), es de la opinión de que "de acuerdo con la impresión general" debe incluirse junto con el xinca en el macromaya, división del macropenucio. En 1950, mantiene esta última posición, si bien menciona de nuevo que se le han señalado afinidades macrochibchas.

Johnson (1940: 89) incluye la familia lenca en el subfilo penucio división, a su vez, del macrofilo penucio.

Jijón y Caamaño (1943: 11), siguiendo a Schmidt, incluye la familia lenca dentro del grupo misquito-xinca (junto con las lenguas misumalpas, el paya, el jicaque y el xinca), que es una de las ocho divisiones que establece del filo macrochibcha.

Loukotka (1948: 196) considera las lenguas lencas como intermediarias entre las chibchas y las mayas.

McQuown (1954: 529) incluye nuevamente el lenca dentro de las lenguas centroamericanas no clasificadas. 
Swadesh (1958: 133-134) agrupa al lenca con el xinca y considera que el jicaque y el filo macrochibcha son las relaciones externas más cercanas. En 1959 (p. 17), denomina al grupo "xilenca" y propone una separación interna de 30 siglos mínimos. En 1967 (pp. 98-99), denomina al grupo "lenca-xinca" y divide al lenca en lenca (lenca hondureño) y chilanga (lenca salvadoreño) entre los que habría 20 siglos mínimos de separación. Considera que existe relación con el nahuatl, con el cual el grupo presenta una divergencia de $\mathbf{4 5}$ siglos mínimos.

Tax (1960: 432) incluye al lenca como grupo aislado dentro del filo macrochibcha.

Greenberg (1960: 793) sitúa al lenca junto con el misumalpa, el paya y el xinca en la segunda de las tres subdivisiones que establece en el subfilo chibcha del filo macrochibcha.

Voegelin y Voegelin (1965: 32) incluyen al lenca como una de las 17 divisiones del filo macrochibcha, clasificándolo como "language isolate".

Holt y Bright (1976: 151) consideran al lenca como grupo aislado sin relación con ninguno otro.

Campbell (1979: 939-940) también trata al lenca como grupo aislado y rebate su pretendida relación con el xinca (1979: 961-962).

De todas las relaciones que se han planteado ente el lenca y otros grupos lingüísticos, sólo se ha intentado aducir indicios en el caso de xinca (Lehmann, 1920: 767), la familia maya (Andrews y Wyllys, 1970) y la familia utoazteca (Escalante y Faier, 1959). Kaufman (1974: 36) señala, no obstante, que "ninguna de estas conjeturas ha sido demostrada como probable".

\subsection{Estudios sobre la relación de las lenguas lencas entre.sí}

Los únicos estudios publicados sobre las relaciones de las lenguas lencas entre sí son los de Campbell $(1976 \mathrm{~b}, 1979)$ en que se señalan 11 correspondencias fonéticas y se incluyen 49 conjuntos de cognados. En su obra de 1979 (p. 941), Campbell informa que Kaufman había reunido cerca de 75 conjuntos de cognados en un trabajo que ha permanecido sin publicarse.

Hasta el momento no ha habido, pues, ninguna aplicación a fondo de los métodos de la lingüística histórica a la relación entre el lenca hondureño y el lenca salvadoreño.

\subsection{Propósito de la investigación}

El objetivo de esta investigación es la reconstrucción de los fonemas segmentales del protolenca con base en la comparación de los materiales disponibles del lenca hondureño y del lenca salvadoreño.

Además del aporte que toda reconstrucción representa en sí para la lingüística diacrónica en general, ésta permitirá contar con una mejor base para el estudio de las posibles relaciones de la familia lenca con otras familias lingüísticas del área, como la chibcha por ejemplo.

\subsection{Marco teórico}

El marco teórico empleado en este estudio es el Método Comparativo de la lingüística diacrónica tal y como se describe en detalle, por ejemplo, en las obras de Meillet $(1921,1925)$ o Hoenigswald $(1950,1960,1973)$.

Conviene señalar que en este caso existen limitaciones derivadas de diversas circunstancias que dificultan (aunque ciertamente no impidan) la aplicación del método comparativo. Estas limitaciones son las siguientes:

a) Lo reducido de los materiales que se han conservado de las dos lenguas comparadas y que difícilmente podrán ampliarse por haberse extinguido el lenca salvadoreño y, muy probablemente también, el hondureño. Aunque la separación temporal no sea excesivamente grande (como se señaló, Swadesh la calculaba en 2.000 años), cuando el número de formas disponibles para la comparación apenas se acerca a unas quinientas, el número de cognados que se descubran es necesariamente bajo.

b) La transcripción en la mayor parte de las fuentes es defectuosa. En general, creo que el análisis de los datos del lenca hondureño efectuado en este estudio y el de los del lenca salvadoreño realizado por el Río Urrutia (1985), aclaran la mayor parte de los problemas ocasionados por esta circunstancia, pero siempre hay un residuo de casos en que no se puede tener total seguridad.

c) No se ha efectuado todavía ningún estudio sistemático de la morfología - tanto flexiva como derivativa - de ambas lenguas, lo cual crea en algunos casos dificultades en materia de segmentación. 
1.1.5. Observaciones sobre la realización de los fonemas consonánticos y su transcripción en las fuentes

/p/ se realizaba como oclusiva sorda (en las fuentes $p$ ) en posición inicial de sílaba excepto en el entorno / a a/. En este entorno se daba una realización fricativa sonora ( $b$ en Campbell et alii, 1978 , y $b$ en las otras fuentes). Ante consonante sorda y silencio parece haberse dado una realización fricativa sorda en variación libre con la oclusiva sorda y la fricativa sonora (en Campbell et alii, 1978, $p$; en las fuentes anteriores: $f$ ). Finalmente, después de nasal se daba una realización oclusiva sonora ( $b$ en las fuentes).

/t/ se realizaba como oclusiva dental sorda a inicio de sílaba (en las fuentes $t$ ). Las apariciones de $d$, muy escasas y nunca en oposición con las de $t$, se han interpretado como variantes sonoras facultativas del mismo fonema. Los entornos en que se han observado son los siguientes: ante linde silábico (p.e. ted 'trabajo') e intervocálicamente precedidas y seguidas por/a/ (p.e. shopada 'nube').

$/ \mathrm{k} /$ se realizaba generalmente en posición inicial de palabra como oclusiva sorda (en las fuentes $c$, $q u, k$ ). En posición intervocálica, parece haber alternado una realización oclusiva sonora con una fricativa sonora (en Campbell et alii, 1978, $g$ y $g$ respectivamente; en las demás fuentes: $g$ ). La realización oclusiva sonora se daba también después de nasal. La realización oclusiva sorda, la fricativa sonora y una fricativa sorda ( $j$ en las fuentes anteriores a Campbell et alii, 1978) parecen haber alternado libremente ante consonante y silencio. Las realizaciones fricativas parecen haber variado en el grado de fricción, pues en algunos casos se percibieron y en otros no (por ejemplo: huagtig y guagti alternan como transcripciones de /waktik/ 'caites'). En algunos dialectos la variante oclusiva sonora se daba también en posición inicial de palabra.

$/ \mathrm{m} /$ (escrita $m$ en las fuentes) parece haberse realizado siempre como nasal bilabial sonora.

$/ \mathrm{n} /$ se realizaba como nasal alveolar sonora $(n$ en las fuentes) a principio de sílaba y a final de sílaba ante consonantes no graves. Ante consonantes graves se realizaba como nasal velar ( $\eta$ en Campbell et alii, 1978, y $n$ en las demás fuentes, en este entorno). Las dos variantes parecen haber alternado libremente en posición final de palabra, caso en que las fuentes anteriores a Campbell et alii, 1978, representan la nasal velar como $n g$. /s/ parece haber tenido realizaciones tanto alveolares como palatales y tanto sonoras como sordas en todos los entornos. La realización alveolar sorda es $s$ o $c$ en las fuentes tempranas y $s$ en Campbell et alii (1978). La alveolar sonora es $z$ en las fuentes tempranas y no aparece en Campbell et alii (1978). La palatal sorda es $s h$ en las fuentes tempranas y $\check{s}$ en Campbell et alii (1978). La palatal sonora es $z h$ en las fuentes tempranas, únicas en que aparece. Campbell et alii (1978) detectaron una realización retrofleja sorda ante /1/.

Algunos ejemplos de estas alternancias son los siguientes: guash, guaz 'agua'; zial, shiol 'aguacate'; zegüe, segue 'zarigüeya'; hazha, asha 'cabello'; siri, ciri 'estrella'; shiri, siri, sirigui 'negro'; nepshe, nepze, nefseg 'nariz'.

/1/ parece haberse realizado siempre como aproximante lateral sonora ( $l$ en las fuentes).

$/ \mathrm{r} /$ de realizaba como vibrante múltiple a principio de palabra y después de consonante nasal $(r$ en las fuentes tempranas y $\bar{r}$ en Campbell et alii, 1978) y como vibrante simple en los demás casos ( $\mathrm{r}$ en las fuentes tempranas y $\check{r}$ en Campbell et alii, 1978). En un solo ejemplo de Campbell et alii (1978) se presenta la vibrante múltiple en posición intervocálica.

/w/ en posición inicial de sílaba se realizaba como aproximante labiovelar sonora ( $h u$ en las fuentes tempranas, $w$ en Campbell et alii, 1978) o como secuencia de $[\mathrm{g}]$ más dicha aproximante $(\mathrm{gu}$ en las fuentes tempranas, $g w$ en Campbell et alii, 1978). En los demás entornos se daba la realización aproximante solamente ( $u$ en las fuentes tempranas, en estos casos). Ocasionalmente, / w/ aparece transcrita también como $v$.

$/ \mathrm{j} /$ se realizaba como aproximante mediopatal sonora. El símbolo empleado en Campbell et alii (1978) es $y$; las otras fuentes usan este símbolo en posición inicial de sílaba, $i$ después de consonante perteneciente a la misma sílaba y tanto y como $i$ a final de sílaba.

\subsubsection{Observaciones sobre la realización de los fonemas vocálicos y su transcripción en las fuentes}

Ninguna de las fuentes utiliza otros símbolos que $i, e, a, o, u$ para la transcripción de las vocales.

Todas las vocales parecen haber tenido variantes largas en sílaba abierta; probablemente, en especial si el acento recaía sobre ellas.

Es probable que /i/ tuviera variaciones en el grado de abertura pues se dan vacilaciones en las 
fuentes, como en el caso de huie $\sim$ hue $\sim$ guilli 'seis' (probablemente algo como [wi·] con una vocal larga de abertura creciente; compárese el lenca salvádoreño / wi / 'seis'). A esto puede haberse sumado la influencia del castellano que tiende a no permitir la presencia de [i] inacentuada a final de palabra, lo cual explicaría desacuerdos como el que se da en el caso de aguingui $\sim$ ahuingui $\sim{ }^{2} a$ winge 'venado'.

/a/ debe haber tenido realizaciones posteriorizadas, pues en varios casos se observan alternancias entre $a$ y $o$ en las transcripciones de las fuentes tempranas: mana $\sim$ mano 'espina'; siga $\sim$ sigo 'verde'; zial shiol 'aguacate'.

Seguida por $/ \mathrm{j} /$ parece haber tenido realizaciones anteriorizadas, según lo indican las alternancias del siguiente tipo en las transcripciones de las fuentes tempranas: tai- $\sim$ tei- 'cortar', guay $\sim$ guey 'llorón'.

Finalmente, ante las vocales iniciales de palabra se insertaba una oclusión glotal que las fuentes tempranas representan ocasionalmente por medio de $h$ y que Campbell et alii (1978) transcriben con el símbolo fonético estándar ( [?] ).

\subsection{Lenca salvadoreño}

De acuerdo con del Río Urrutia (1985: 12-27), los fonemas segmentales del lenca salvadoreño son los representados en los cuadros que se presentan a continuación.

\subsubsection{Consonantes}

Dental/

\begin{tabular}{|c|c|c|c|c|c|}
\hline & & Dental/ & & & \\
\hline Nasales & $\begin{array}{c}\text { Bilabial } \\
\mathrm{m}\end{array}$ & $\begin{array}{c}\text { Alveolar } \\
n\end{array}$ & Palatal & Velar & Laríngea \\
\hline $\begin{array}{l}\text { Oclusivas } \\
\text { simples }\end{array}$ & $\mathrm{p}$ & $\mathrm{t}$ & & $\mathrm{k}$ & \\
\hline $\begin{array}{l}\text { Oclusivas } \\
\text { glotalizadas }\end{array}$ & $\mathrm{p}^{\prime}$ & $t^{\prime}$ & & $\mathrm{k}^{\prime}$ & \\
\hline Africadas & & ts' & $\mathrm{t} \int$ & & \\
\hline Fricativas & & s & $\int$ & & $\mathrm{h}$ \\
\hline Lateral & & 1 & & & \\
\hline Vibrante & & $\mathrm{r}$ & & & \\
\hline Semiconsonantes & w & & $\mathrm{j}$ & & \\
\hline
\end{tabular}
[x].

1.2.2. Vocales

$\begin{array}{lccc} & \text { Anterior } & \text { Central } & \text { Posterior } \\ \text { Alta } & \mathrm{i} & & \mathrm{u} \\ \text { Media } & \mathrm{e} & & \mathrm{o}\end{array}$

Baja

a

\subsubsection{Observaciones}

Como en el estudio citado de del Río Urrutia (1985) se hace una descripción pormenorizada del sistema fonológico del lenca salvadoreño, aquí me limitaré a señalar que se han seguido en todos los casos sus criterios y su transcripción, excepto en el uso de /N/ para representar las apariciones de [ $\mathrm{g}$ ] en los casos en que no estaba claro si procedía de $/ \mathrm{n} / \mathrm{o} / \mathrm{m} /$, fonemas que estaban en proceso de neutralizarse a final de sílaba en la época en que sus fuentes recogieron los datos. La comparación con el lenca hondureño permite resolver, en las formas tomadas en cuenta para este estudio, dicha ambigüedad. Por otra parte, se han reemplazado sus símbolos / ¿'/ y / č'/ por / ts'/ y / t f'/.

En cuanto a realizaciones de los fonemas, tiene interés señalar que - de acuerdo con del Río Urrutia- $[\mathrm{p}],[\mathrm{t}],[\mathrm{k}],[\mathrm{s}]$ y $\left[\int\right]$ presentan variantes sonoras en contacto con consonantes sonoras y entre vocales.

/ k'/ en posición intervocálica presenta la oclusión glotal como alófono facultativo de [k'].

$/ \mathrm{h} / \mathrm{se}$ realiza tanto por medio de $[\mathrm{h}]$ como de

$/ \mathrm{r} /$ tiene realizaciones tanto simples como múltiples (estas últimas con frecuencia ensordecidas). 


\section{Sistema de fonemas segmentales recons- 2.1.1. Vocales truido para el protolenca}

Como convención en este capítulo al comparar formas de una y otra lengua, la primera siempre será la del lenca hondureño y la segunda, del salvadoreño.

2.1. Cuadros de los fonemas segmentales del protolenca

$\begin{array}{lccc} & \text { Anterior } & \text { Central } & \text { Posterior } \\ \text { Alta } & \mathrm{i} & & \mathrm{u} \\ \text { Media } & \mathrm{e} & & \mathrm{o} \\ \text { Baja } & & \mathrm{a} & \end{array}$

\subsubsection{Consonantes}

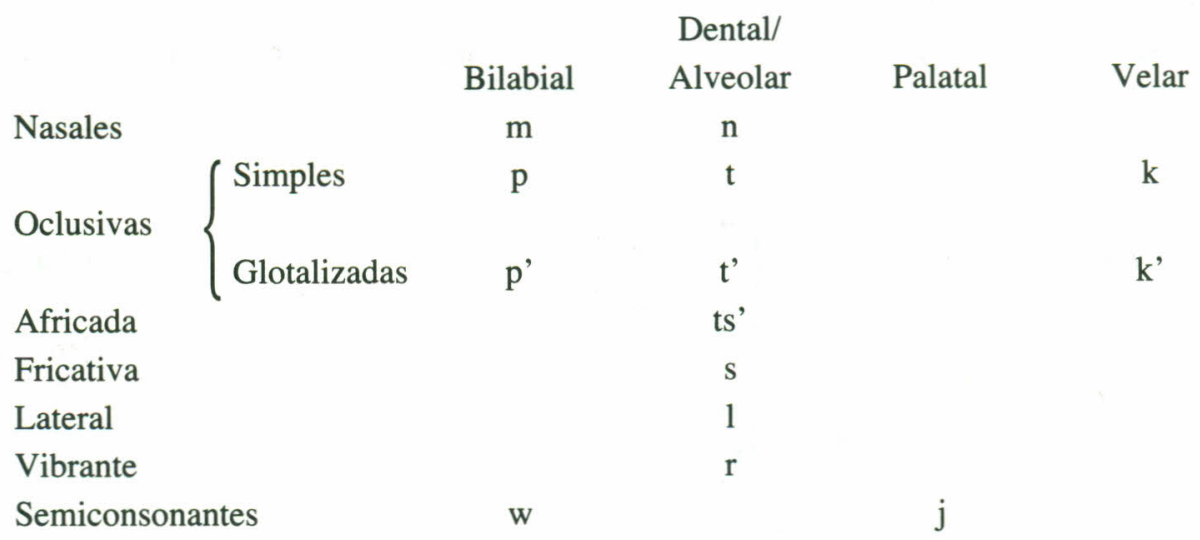

2.2. Cuadros de los reflejos de los protofonemas en las lenguas descendientes.

Símbolos especiales usados en los cuadros o.e. otros entornos

\# linde de palabra

$\$$ linde silábico

2.2.1. Cuadros de los reflejos de las protoconsonantes

\begin{tabular}{|c|c|c|}
\hline Protolenca & $*_{m}$ & $* n$ \\
\hline Lenca hondureño & $\begin{array}{l}ø / \mathrm{a} \ldots \# \\
\text { m(o.e. })\end{array}$ & $\begin{array}{l}\varnothing \sim \mathrm{n} / \mathrm{na} \text { _\# } \\
\mathrm{n}(\mathrm{o} . \mathrm{e} .)\end{array}$ \\
\hline Lenca salvadoreño & $m$ & $\begin{array}{l}ø / \text { la } \_\# \\
\text { n (o.e.) }\end{array}$ \\
\hline
\end{tabular}




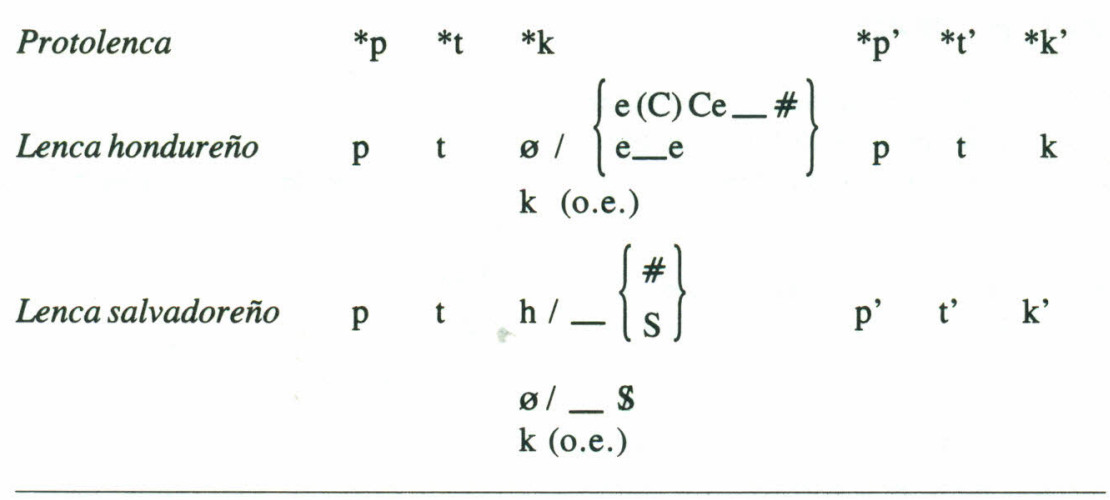

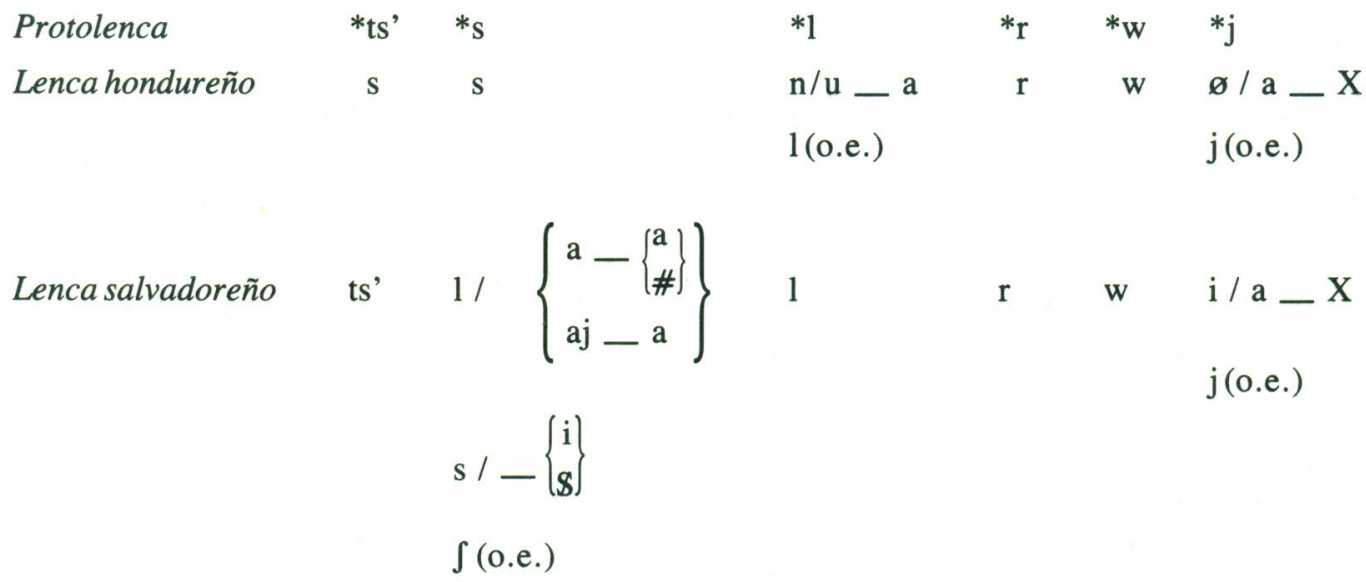

2.2.2. Cuadro de los reflejos de las protovocales (excluidos los casos de pérdida)

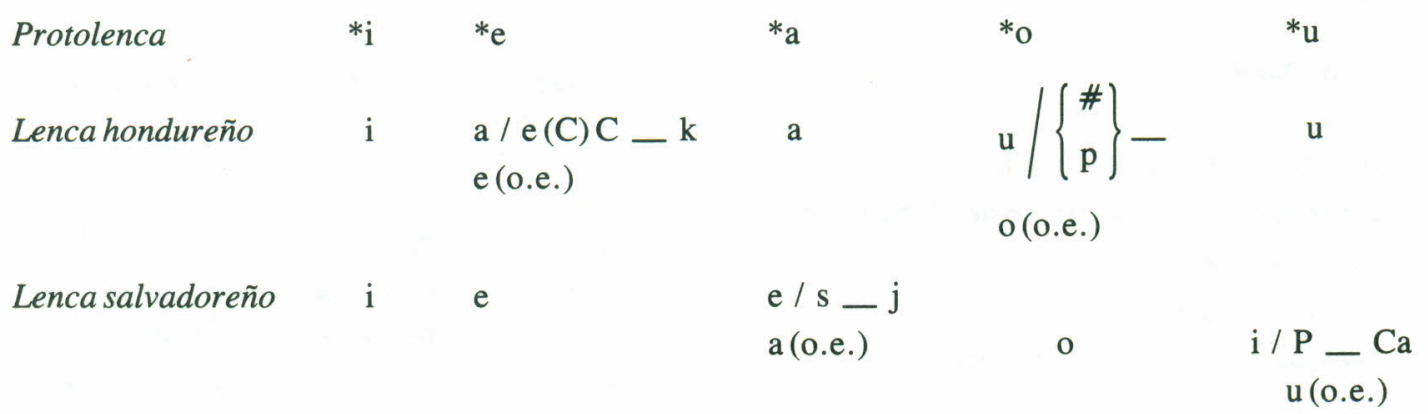


2.3. Ejemplos de los reflejos de los protofonemas consonánticos

\subsection{1. $* / \mathrm{m} /$}

Se dan dos conjuntos de cognados en que $* / \mathrm{m} /$ precedida por $* / a /$ cae ante linde de palabra en el lenca hondureño:

\begin{tabular}{|c|c|c|}
\hline kuma & kumam & 'uña’ \\
\hline & $\int \mathrm{am}$ & 'bueno' \\
\hline \multicolumn{3}{|c|}{ En los demás entornos, ambas lenguas presentan $/ \mathrm{m} /$} \\
\hline mal & & 'roble' \\
\hline musu & muts'u & ‘hígado’ \\
\hline lamta 'refajado' & lamtara & 'ropa' \\
\hline lum- & lum- & 'mover' \\
\hline
\end{tabular}

\subsection{2. */n/}

En el lenca hondureño se da caída, al parecer facultativa de $* / \mathrm{n} /$ precedida por $* / \mathrm{a} /$ ante linde de palabra. En los tres casos observados, la $* / \mathrm{n} /$ está precedida por una secuencia de $* / \mathrm{na} /$ :

$\begin{array}{lll}\text { amnan } \sim \text { amna } & \text { manani } & \text { 'tú' } \\ \text { inan } \sim \text { ina } & \text { inani } & \text { 'él' } \\ \text { unan } \sim \text { una } & \text { unani } & \text { 'yo' }\end{array}$

Hay dos casos en que el lenca salvadoreño presenta $\varnothing$ en la posición en que el lenca hondureño presenta $/ \mathrm{n} /$. El factor condicionante parece ser la presencia de la secuencia /la/ $(<* / \mathrm{la} / \mathrm{o} * / \mathrm{sa} /)$; probablemente se trate de una disimilación eliminatoria de la /n/por la /1/:

$\begin{array}{lll}\text { wasan } & \text { wila } & \text { 'orina' } \\ \text { kunan } & \text { k'ula } & \text { 'quién' }\end{array}$
/n/:

En los demás entornos, ambas lenguas presentan

$\begin{array}{lll}\text { nek } & \text { neh } & \text { 'diente' } \\ \text { na 'este' } & \text {-na } & \text { 'artículo definido' } \\ \text { kin } & \text { k'in } & \text { 'camino' } \\ \text { en- } & \text { en- } & \text { 'oír' } \\ \text { un- } & \text { ona- } & \text { 'estar enfermo' }\end{array}$

Un único conjunto de cognados parece presentar en el lenca hondureño pérdida de $* / \mathrm{n} /$ a final de sílaba interna:

\subsection{3.*/p/}

*/p/ presenta /p/ como reflejo en ambas lenguas:

$\begin{array}{lll}\text { pe } & \text { pe } & \text { 'dos' } \\ \text { lepa } & \text { lepa } & \text { 'jaguar' } \\ \text { pu- } & \text { po- } & \text { 'venir' } \\ \text { apinan } & \text { pinani } & \text { 'nosotros' } \\ \text { nepsek } & \text { nepkuru } & \text { 'nariz' }\end{array}$

2.3.4. $* / \mathrm{t} /$

$* / t /$ presenta / $/$ como reflejo en ambas lenguas:

$\begin{array}{lll}\text { ta } & \text { ta } & \text { 'milpa' } \\ \text { tem } & \text { tem } & \text { 'piojo' } \\ \text { twa- } & \text { twa- } & \text { 'bañar' } \\ \text { waktik } & \text { watih } & \text { 'caites' } \\ \text { katu } & \text { katu } & \text { 'araña' }\end{array}$

\subsection{5. $* / \mathrm{k} /$}

En posición inicial de sílaba, el reflejo de $* / \mathrm{k} /$ es $/ \mathrm{k} /$ en ambas lenguas:

$\begin{array}{lll}\text { kotan } & \text { kotan } & \text { 'montaña' } \\ \text { ke } & \text { ke } & \text { 'piedra' } \\ \text { katu } & \text { katu } & \text { 'araña' } \\ \text { kuma } & \text { kumam } & \text { 'uña' } \\ \text { temaskin } & \text { tenmaskin } & \text { 'peine' } \\ \text { juku } & \text { juku } & \text { 'coyol' }\end{array}$

Hay, sin embargo, conjuntos de cognados que presentan pérdida de $* / \mathrm{k} /$ precedida y seguida por */e/ y en el entorno e (C) $\mathrm{Ce} \_$\# en el lenca hondureño:

$\begin{array}{lll}\text { tet } & \text { teket } & \text { 'trabajar' } \\ \text { pela } & \text { peleh } & \text { 'hermano' }(<* \text { pelek } /) \\ \text { texta } & \text { teteh } & \text { 'anciana' (probable- } \\ & & \text { mente }<* / \text { tektek/) }\end{array}$

En posición final de sílaba interna, se da pérdida de $* / \mathrm{k}$ / en lenca salvadoreño, excepto ante semiconsonante:

\begin{tabular}{|c|c|c|}
\hline waktik & watih & ‘caites’ \\
\hline kakma & k'ama & 'guacal' \\
\hline siksik & sisih & $\begin{array}{l}\text { "camarón (véase este ru- } \\
\text { bro en la lista de conjun- } \\
\text { tos de cognados) }\end{array}$ \\
\hline
\end{tabular}


En posición final de palabra, lo mismo que al final de sílaba interna si sigue una semiconsonante, $* / \mathrm{k} /</ \mathrm{h} /$ en lenca salvadoreño.

$\begin{array}{lll}\text { ikwe } & \text { ihwa } & \text { 'cantar' } \\ \text { nek } & \text { neh } & \text { 'diente' } \\ \text { insek } & \text { ints'eh } & \text { 'pico' } \\ \text { waktik } & \text { watih } & \text { 'caites' }\end{array}$

Del Río Urrutia (1985: 19-20) señala que en el lenca salvadoreño $/ \mathrm{h} / \mathrm{y} / \mathrm{k} /$ coinciden en los mismos entornos en muy pocos casos.

\subsection{6. */p'/}

Este segmento se reconstruye tentativamente en vista de que por el momento se conoce un único conjunto de cognados que apoye su reconstrucción:

napir map'it 'puerco montés'

La existencia de este protofonema sería, por una parte, de esperarse, por razones de simetría, ya que hay indicios más fuertes para reconstruir $* / \mathrm{t}^{\prime} / \mathrm{y}$ pruebas muy claras para $* / \mathrm{k}^{\prime} / \mathrm{y} * / \mathrm{ts} \%$. Por otra parte, llama la atención la frecuencia muy baja de /p'/ en la lengua que la presenta: el lenca salvadoreño.

\subsection{7.*/t'/}

El reflejo de $* / t^{\prime} /$ en el lenca salvadoreño es $/ t ' / y$, en el hondureño, $/ t /$ :

$\begin{array}{lll}\text { taw } & \text { t'aw } & \text { 'casa' } \\ \text { tutu } & \text { t'ut'u } & \text { 'pulga' }\end{array}$

\subsection{8. */k'/}

El reflejo de $* / \mathrm{k}^{\prime} /$ en el lenca salvadoreño es $/ \mathrm{k}^{\prime} / \mathrm{y}$, en el hondureño, $/ \mathrm{k} /$ :

$\begin{array}{lll}\text { kakma } & \text { k'ama } & \text { 'guacal' } \\ \text { kin } & \text { k'in } & \text { 'camino' } \\ \text { kunan } & \text { k'ula } & \text { 'quién' } \\ \text { kelkin } & \text { k'elkin } & \text { 'comal' } \\ \text { juka } & \text { ik'an } & \text { 'fuego' }\end{array}$

\subsection{9. $* /$ ts'/ $^{\prime}$}

El reflejo de $* /$ ts'/ en el lenca salvadoreño es /ts'/ y, en el hondureño, /s/:

$\begin{array}{lll}\text { sewe } & \text { ts'ewe } & \text { 'zarigüeya' } \\ \text { saj } & \text { ts'aj } & \text { 'cinco' } \\ \text { suj- } & \text { ts'u- } & \text { 'chupar' } \\ \text { sisi } & \text { its'its'i } & \text { 'hormiga' } \\ \text { musu } & \text { muts'u } & \text { 'hígado' }\end{array}$

2.3.10. */s/

El reflejo de $* / \mathrm{s} /$ en el lenca hondureño es /s/. En el lenca salvadoreño es $/ 1 /$ cuando está prec $€$ dido por $* / a /$ y seguido por $* / a /$ o silencio y cuando está entre $* / a j / y * / a / ; / s /$ delante de $* / i /$ y linde silábico; y / / / en los demás entornos:

$\begin{array}{lll}\text { was } & \text { wal } & \text { 'agua' } \\ \text { asa } & \text { alah } & \text { 'cabello' } \\ \text { wasan } & \text { wila } & \text { 'orina' } \\ \text { siksik } & \text { sisih } & \text { 'camarón' } \\ \text { siri } & \text { sirih } & \text { 'estrella' } \\ \text { sili } & \text { sili } & \text { 'quebracho' } \\ \text { temaskin } & \text { tenmaskin } & \text { 'peine' } \\ \text { kus } & \text { kuskus } & \text { 'zopilote' } \\ \text { surisur } & \text { Jurih } & \text { 'ardilla' } \\ \text { saj- } & \text { Jej- } & \text { 'desear' } \\ \text { suna } & \text { fila } & \text { 'flor' } \\ \text { so } & \text { Jo } & \text { 'lluvia' }\end{array}$

No obstante lo dicho, un conjunto de cognados muestra /r/ como aparente reflejo de $* / \mathrm{s} /$ en posición intervocálica después de $* / \mathrm{i} /$ en lenca hondureño:

ira- isa- 'sembrar'

\subsubsection{1. */1/}

El reflejo de $* / 1 /$ en lenca hondureño es $/ \mathrm{n} /$ entre $* / \mathrm{u} / \mathrm{y} * / \mathrm{a} /, \mathrm{y} / 1 /$ en los demás casos. En lenca salvadoreño, es siempre $/ 1 /$.

$\begin{array}{lll}\text { lawa } & \text { lawa } & \text { 'tres' } \\ \text { liwa- } & \text { liwa- } & \text { 'comprar' } \\ \text { lepa } & \text { lepa } & \text { 'jaguar' } \\ \text { lum- } & \text { lum- } & \text { 'mover' } \\ \text { wala } & \text { wala } & \text { 'mapachín' } \\ \text { sili } & \text { sili } & \text { 'quebracho' } \\ \text { tal- } & \text { tal- } & \text { 'beber' }\end{array}$


kelkin

kunan

suna

$\begin{array}{ll}\text { k'elkin } & \text { 'comal' } \\ \text { k'ula } & \text { 'quién' } \\ \text { Jila } & \text { 'flor' }\end{array}$

\subsubsection{2. */r/}

El reflejo de $* / \mathrm{r} /$ en ambas lenguas es $/ \mathrm{r} /$ :

$\begin{array}{lll}\text { siri } & \text { sirih } & \text { 'estrella' } \\ \text { wara } & \text { wara } & \text { 'río' } \\ \text { murak } & \text { muraka } & \text { 'jocote' } \\ \text { surisur } & \text { Jurih } & \text { 'ardilla' }\end{array}$

\subsubsection{3.*/w/}

El reflejo de $* / w /$ en ambas lenguas es /w/:

$\begin{array}{lll}\text { wewe } & \text { wewe } & \text { 'niño' } \\ \text { wara } & \text { wara } & \text { 'río' } \\ \text { sewe } & \text { ts'ewe } & \text { 'zarigüeya' } \\ \text { lawa } & \text { lawa } & \text { 'tres' } \\ \text { twa- } & \text { twa- } & \text { 'bañar' }\end{array}$

\subsubsection{4. $* / \mathrm{j} /$}

En el lenca hondureño, $* / \mathrm{j} /$ se pierde cuando está precedida por $* / a /$ y no está seguida por linde de morfema. En los demás casos el reflejo es $/ \mathrm{j} /$.

En el lenca salvadoreño, el reflejo es / i/ cuando está precedida por $* / a / y$ no está seguida por linde de morfema (caso en que $* / a /$ cae); en los demás casos es $/ \mathrm{j} /$ :

$\begin{array}{lll}\text { juku } & \text { juku } & \text { 'coyol' } \\ \text { jol- } & \text { jolo- } & \text { 'reír' } \\ \text { jet- } & \text { jete- } & \text { 'tocar' } \\ \text { saj } & \text { ts'aj } & \text { 'cinco' } \\ \text { wasa- } & \text { wila- } & \text { 'orinar' (*wajsa-) } \\ \text { ama } & \text { ima } & \text { 'maíz' (*ajma) } \\ \text { sak- } & \text { ts'ih- } & \text { 'lavar' (*ts'ajk-) }\end{array}$

No obstante, hay un caso en que $* / \mathrm{j} /$ parece haber caído en el lenca salvadoreño ante / $\mathrm{i} /$ derivada de $* / \mathrm{u} /$ (véase 2.4.6.):

$$
\text { juka ik'an 'fuego' }
$$

En dos conjuntos de cognados $/ \mathrm{j} /$ del lenca hondureño se ve correspondida por $\varnothing$ en el lenca salvadoreño. Es probable que el fenómeno en estos casos no sea fonológico sino que la /-j/ del lenca hondureño sea un formativo de temas; en uno de los conjuntos, este elemento aparece unido a la raíz en un tema que en el lenca salvadoreño se forma por medio de reduplicación:

$$
\begin{array}{lll}
\text { suj } & \text { Jufu } & \text { 'perro' } \\
\text { suaj } & \text { Jua } & \text { 'coyote' }
\end{array}
$$

2.4. Ejemplos de los reflejos de los protofonemas vocálicos

En 2.4.1. se tratan los casos de correspondencias de vocales con ø; en las siguientes secciones, las correspondencias entre vocales.

\subsubsection{Correspondencias de vocales con $\varnothing$}

\subsubsection{Casos de síncopa cuando preceden */1/ o consonantes nasales}

En una serie de morfemas disilábicos que no funcionan independientemente como palabras, la vocal final se pierde ante un morfema siguiente

\begin{tabular}{|c|c|c|}
\hline amnan & manani & $\begin{array}{l}\text { 'tú' ('LH -nan, LS -nani } \\
\text { 'formativo de temas } \\
\text { pronominales') }\end{array}$ \\
\hline jol- & jolo- & 'reír' \\
\hline & eni- & ‘oír’' \\
\hline & uli- & 'bailar' \\
\hline & tali- & 'beber' \\
\hline & $\begin{array}{l}\text { ona- } \\
\text { ts'ana- }\end{array}$ & $\begin{array}{l}\text { 'estar enfermo' } \\
\text { 'hcer frío' }\end{array}$ \\
\hline
\end{tabular}
cuando está precedida por consonante nasal o*/1/:

Cuando la vocal final es */i/, en el lenca salvadoreño también se da la caída en entornos que todavía no se han determinado, de modo que / eni-/ alterna con /en-/, /uli-/ con /ul-/, etc.

Un conjunto -LH jet-, LS jete- sugiere que la caída pudiera haberse dado después de cualquier consonante de la serie dental/alveolar.

\subsubsection{Síncopa o aféresis de */a/ en el lenca} salvadoreño ante $* / \mathrm{j} /$.

$* / \mathrm{a} /$ cae ante $* / \mathrm{j} /$ en el lenca salvadoreño cuando no está seguida la secuencia por un linde morfemático (véase 2.3.14.). La selección de */aj/ como reconstrucción de la correspondencia $\mathrm{LH} / \mathrm{a} /$, LS /i/ se hizo con base en que al conjunto de 
cognados LH / ama/ -LS / ima/ corresponden en otras lenguas centroamericanas formas con el diptongo /aj-/ (p.e.: cacaopera /ajma/ 'maíz').

$\begin{array}{lll}\text { aj- } & \text { aj- } & \text { 'decir' } \\ \text { saj } & \text { ts'aj } & \text { 'cinco' } \\ \text { wasan- } & \text { wila- } & \text { 'orinar' } \\ \text { ama } & \text { ima } & \text { 'maíz' } \\ \text { sak- } & \text { ts'ih- } & \text { 'lavar' }\end{array}$

2.4.1.3. Apócope de $* /$ i/ final de palabra en el lenca hondureño

*/i/ cae en el lenca hondureño ante linde de palabra cuando está precedida por $* / \mathbf{n} /$. Nótese que todas las formas observadas son pronombres personales terminados en el formativo*/-nani/.

$\begin{array}{lll}\text { unan } & \text { unani } & \text { 'yo' } \\ \text { amnan } & \text { manani } & \text { 'tú' } \\ \text { apinan } & \text { pinani } & \text { 'nosotros' } \\ \text { inan } & \text { inani } & \text { 'él' }\end{array}$

2.4.1.4. Aféresis de $* / a /$ en el lenca salvadoreño

*/a/ cae en el lenca salvadoreño cuando está precedida por linde de palabra y seguida por consonante bilabial:

apinan pinani 'nosotros'

amnan manani 'tú' $(<* /$ amanani/ $)$

\subsection{2. */i/. Correspondencia LH /i/ - LS /i/}

$\begin{array}{lll}\begin{array}{l}\text { sili } \\ \text { in }\end{array} & \begin{array}{l}\text { sili } \\ \text { ints'ats'a }\end{array} & \begin{array}{l}\text { 'quebracho' } \\ \text { 'boca' }\end{array} \\ \text { kelkin } & \text { k'elkin } & \text { 'comal' } \\ \text { insek } & \text { ints'eh } & \text { 'pico' } \\ \text { kin } & \text { k'in } & \text { 'camino' } \\ \text { liwa- } & \text { liwa- } & \text { 'comprar' } \\ \text { apinan } & \text { pinani } & \text { 'nosotros' }\end{array}$

2.4.3. */e/. Correspondecias LH /a/ -LS /e/, $\mathrm{LH} / \mathrm{e} / \mathrm{-LS} / \mathrm{e} /$

En dos conjuntos de cognados */e/ seguida por $* / \mathrm{k}$ / final de palabra parece haberse disimilado en /a/ de una $* / \mathrm{e} /$ precedente:

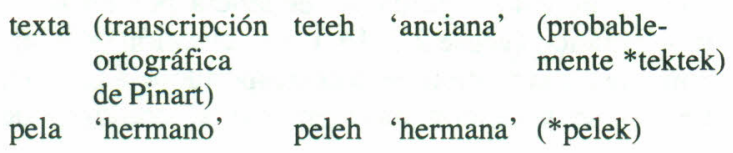

En los demás casos, el reflejo de */e/ en ambas lenguas es /e/.

$\begin{array}{lll}\text { ke } & \text { ke } & \text { 'piedra' } \\ \text { en- } & \text { eni- } & \text { 'oír' } \\ \text { nek } & \text { neh } & \text { 'diente' } \\ \text { sewe } & \text { ts'ewe } & \text { 'zarigüeya' } \\ \text { kelkin } & \text { k'elkin } & \text { 'comal' } \\ \text { wewe } & \text { wewe } & \text { 'niño' } \\ \text { insek } & \text { ints'eh } & \text { 'pico' }\end{array}$

\subsection{4. */a/. Correspondencia LH /a/ - LS /a/}

$\begin{array}{lll}\text { ta } & \text { ta } & \text { 'milpa' } \\ \text { wara } & \text { wara } & \text { 'río' } \\ \text { kunan } & \text { k'ula } & \text { 'quién' } \\ \text { mal } & \text { mal } & \text { 'roble' } \\ \text { aj- } & \text { aj- } & \text { 'decir' } \\ \text { Kakma } & \text { k'ama } & \text { 'guacal' }\end{array}$

Un conjunto muestra la correspondencia $\mathrm{LH} / \mathrm{a} /$ - LS /e/ ante /j/. El cambio de */a/ a /e/ podría haber sido favorecido por ser LS $/ \int /(<* / \mathrm{s} /)$ la consonante precedente:

$$
\text { saj- Jej- 'desear' }
$$

\subsection{5. */o/. Correspondencias $\mathrm{LH} / \mathrm{u} /-\mathrm{LS} / \mathrm{o} /$, $\mathrm{LH} / \mathrm{o} / \mathrm{LS} / \mathrm{o} /$}

Tres conjuntos de cognados muestran la correspondencia LH/u/-LS /o/. En dos de los casos, el entorno es la posición inicial de palabra. En el otro, precede $* / \mathrm{p} /$. Esta correspondencia se ha asignado a $* / o /$ en vista de que la correspondencia $\mathrm{LH} / \mathrm{u} /$ -LS $/ \mathrm{u} /(<* / \mathrm{u} /)$ se da en los mismos entornos, en tanto la correspondencia $\mathrm{LH} / \mathrm{o} / \mathrm{-LS} / \mathrm{o} /(<* / \mathrm{o} /)$ no se presenta en ellos.

$\begin{array}{lll}\text { pu- } & \text { po- } & \text { 'venir' } \\ \text { u- } & \text { o- } & \text { 'ir' } \\ \text { un- } & \text { ona- } & \text { 'estar enfermo' }\end{array}$

En los demás casos, /o/ es el reflejo de $* / 0 /$ en ambas lenguas:

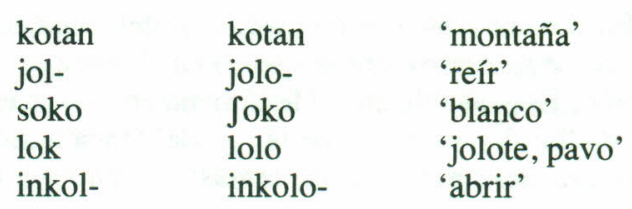




\subsection{6. */u/. Correspondencias LH /u/ -LS /i/, $\mathrm{LH} / \mathrm{u} / \mathrm{-LS} / \mathrm{u} /$}

En dos conjuntos de cognados, el fonema / $\mathrm{u} /$ del lenca hondureño se presenta en correspondencia con el fonema / i/ del lenca salvadoreño. En ambos casos, estos fonemas están precedidos por una consonante continua no grave y seguidos por una sílaba cuyo núcleo es $* / a /$ :

$\begin{array}{lll}\text { suna } & \text { Sila } & \text { 'flor' } \\ \text { juka } & \text { ik'an } & \text { 'fuego' }\end{array}$

La entidad reconstruida con base en la correspondencia $\mathrm{LH} / \mathrm{u} / \mathrm{-LS} / \mathrm{i} /$ ha sido */u/ en vista de que fue en la secuencia $* /$ ula/ que $* / 1 /$ dio $/ \mathrm{n} /$ como resultado en el lenca hondureño.

En los demás entornos, el reflejo de $* / \mathrm{u} /$ es $/ \mathrm{u} /$ en ambas lenguas:

$\begin{array}{lll}\text { lum- } & \text { lum- } & \text { 'mover' } \\ \text { juku } & \text { juku } & \text { 'coyol' } \\ \text { tutu } & \text { t'ut'u } & \text { 'pulga' } \\ \text { kuma } & \text { kuman } & \text { 'uña' } \\ \text { musu } & \text { muts'u } & \text { 'hígado' } \\ \text { katu } & \text { katu } & \text { 'araña' } \\ \text { unan } & \text { unani } & \text { 'yo' }\end{array}$

\section{Conjuntos de cognados}

A continuación se incluye la lista de conjuntos de cognados en orden alfabético según las glosas castellanas.

Con la finalidad de lograr un máximo de explicitez en cuanto a las razones para considerar las formas como cognadas, se discute y se justifica la segmentación propuesta siempre que esto parece necesario. En los casos en que el conocimiento existente de la morfología de las lenguas no permite identificar algún segmento, éste se glosa con '?'.

En una mayoría de casos se ofrece la protoforma reconstruida; no obstante, hay algunos conjuntos en que esto no es posible debido a la presencia en ellos de emparejamientos únicos.

Como sucede con frecuencia (Meillet, 1925:39), el número de conjuntos de cognados es bastante restringido. En el caso particular de este estudio, esto puede atribuirse a las limitaciones de los materiales disponibles y a la profundidad de la separación entre las dos lenguas.

Las formas citadas, en el caso de sustantivos, adjetivos y verbos, son los temas desprovistos de afijos flexivos.
Finalmente, el significado de la forma de una lengua se cita a su lado cuando no corresponde al de la glosa con que se presenta el rubro en cuestión.

Abreviaciones citadas en los conjuntos de cognados:

OP: Vocabulario del lenca de Opatoro de E.G. Squier

GM: Vocabulario del lenca de Guajiquiro de
A. Membreño

SI: Vocabulario del lenca de Cabañas (Similatón de A. Membreño

GP: Vocabulario del lenca de Guajiquiro de A.L. Pinart

CChD: Vocabulario del lenca de Intibucá de Campbell, Chapman y Dakin

GS: Vocabulario del lenca de Guajiquiro de E.G. Squier

1. abrir

*inkolo-

LH inkolingol- $(\mathrm{GM}, \mathrm{SI})$

LS inkolo-

2. agua

$$
\begin{aligned}
& \text { LH was } \\
& \text { guash }(\mathrm{GM}, \mathrm{SI}) \text { guaz }(\mathrm{GP})
\end{aligned}
$$

3. anciana

LH texta (GP)

LS teteh

La forma del lenca hondureño se transcribe como aparece en la lista de Pinart tomada en Guajiquiro, pues se desconoce qué sonido se representó con la $x$ en ella. Podría haberse tratado de una $/ \mathrm{k} /$, caso en que se reconstruiría *tektek.

$\begin{array}{llll}\text { 4. } & \begin{array}{l}\text { araña } \\ { }_{\text {*katu }}\end{array} & \begin{array}{l}\text { LH katu } \\ \text { cátu (GM, GP) }\end{array} & \text { LS katu } \\ \text { 5. } & \begin{array}{l}\text { ardilla } \\ \text { *suri }\end{array} & \begin{array}{l}\text { LH surisur } \\ \text { shurishur(GP) }\end{array} & \text { LS Jurih }\end{array}$

Este conjunto presenta la dificultad de que la forma del lenca hondureño no tiene la $/ \mathrm{k} /$ que se esperaría como correspondencia de la $/ \mathrm{h} /$ del lenca salvadoreño. Podría pensarse que la $/ \mathrm{k} /$ tïnal de sílaba no hubiera sido percibida por Pinart (como parece haber sucedido en otros casos), pero esto dificultaría la explicación de la pérdida de la /i/ en el segundo miembro de la reduplicación. La reduplicación parece haber funcionado como formativo de temas en ambas lenguas, pero no siempre hay coincidencia en su uso.
6. bailar
LH ul-
LS uli- 


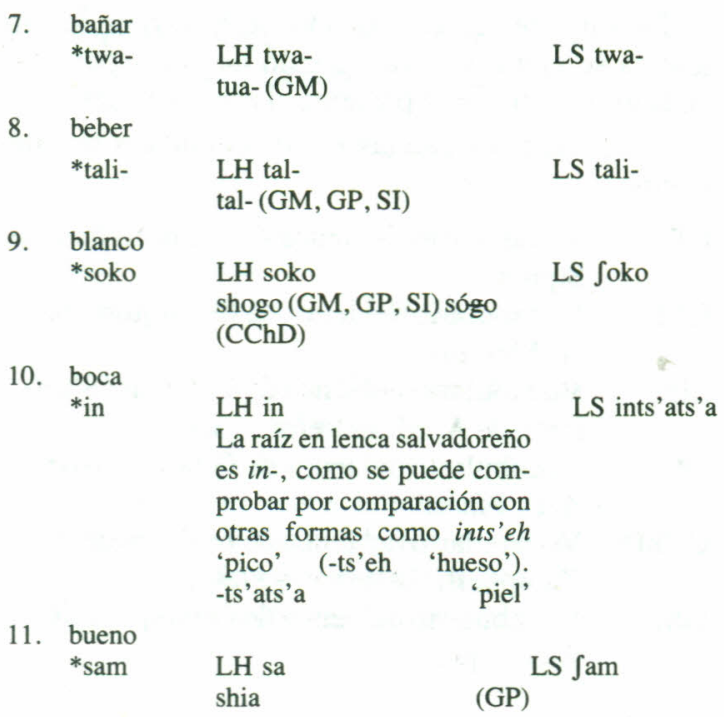

En vista de que no se han encontrado pérdidas de $/ *_{\mathrm{j}} /$ de este tipo en otros casos, parece posible suponer que toda la secuencia shi esté representando la realización palatal de /s/ en el lenca hondureño.

\section{2. cabello *asak}

LH asa
hazga (GP) asha (GM)

LS alah

A la / h/ final del LS debería corresponder $/ \mathrm{k} /$ en el LH, y muy probablemente así fue. La variante final de sílaba de / $\mathrm{k}$ / en esta lengua podía ser muy débil y pasar desapercibida a personas sin entrenamiento en transcripción fonética. Esto se ve con claridad en casos como el de huagtig $\sim$ guagti.

$$
\begin{aligned}
& \text { 13. caites } \\
& \text { *waktik LH waktik LS watih } \\
& \text { huagtig (GM) guagti (SI) } \\
& \begin{array}{l}
\text { 14. camarón } \\
\text { *siksik }
\end{array} \\
& \begin{array}{l}
\text { LH siksik } \\
\text { sigsig (GM) }
\end{array} \\
& \text { LS sisih }
\end{aligned}
$$

La fuente de la forma del lenca salvadoreño es Peccorini (incluido en Lehmann, 1920:695) quien transcribió sisís. Por medio de las formas recogidas por Lehmann (1920), sabemos que /h/ tenía, en lenca salvadoreño, un alófono fricativo dental sordo que variaba libremente con $[\mathrm{x}] \mathrm{y}[\mathrm{h}]$ ante $/ \mathrm{n} /$. Por el acento en la sílaba final, es muy probable que Peccorini recogiera la forma con el sufijo /-na/ 'definido', pero omitiera este último al hacer la lista, ya que el acento de los sustantivos recae siem- pre en la penúltima sílaba. Un ejemplo de la variación de realizaciones de $/ \mathrm{h} /$ es el siguiente:

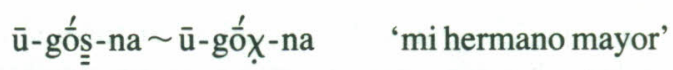

Con base en lo anterior, y en lo que presenta el lenca hondureño, se ha considerado que la $s$ final de la forma sisís representa el fonema /h/, no el fonema /s/ del lenca salvadoreño.

$$
\begin{array}{lll}
\text { 15. } \begin{array}{ll}
\text { camino } \\
\text { *k'in }
\end{array} & \begin{array}{l}
\text { LH kin } \\
\text { quin (GP, SI) }
\end{array} & \text { LS k'in } \\
\text { 16. } & \text { casa } & \\
\text { *t'aw } & \begin{array}{l}
\text { LH taw } \\
\text { thau (GP) taug (GM) tou (SI) }
\end{array} & \text { LS t'aw }
\end{array}
$$

La $g$ final de la forma recogida por Membreño es problemática, pues ni las otras fuentes del lenca hondureño ni el lenca salvadoreño apoyan la idea de que la palabra tuviera una $/ \mathrm{k} /$ final. La $h$ de la forma recogida por Pinart podría hacer pensar en glotalización, pero no hay ningún otro caso que sugiera que ésta se diera en el lenca hondureño. Los datos de Campbell et alii (1978) presentan apariciones de la oclusión glotal antes de las vocales iniciales, pero en ningún caso consonantes glotalizadas.

$$
\begin{aligned}
& \text { 17. cerrar } \\
& \begin{array}{ll}
* \text { inkap- } & \text { LH inkop- } \\
\text { in cop-(GM) cop-(SI) } & \text { LS inkap- }
\end{array}
\end{aligned}
$$

Como su antónimo *inkolo- 'abrir', este elemento parece incluir la raíz *in- 'boca'. La presencia de una $o$ en el lenca hondureño en lugar de la /a/ esperada podría indicar la realización posteriorizada de este fonema aludida en 1.1.6. o podría ser el resultado de un cambio analógico (contaminación con la vocal de *inkolo-).
18. cinco$$
\text { *ts'aj }
$$$$
\text { LH saj }
$$$$
\text { saay (GP) sai (GM) say (SI) }
$$
19. comal
*k'elkin

$$
\begin{aligned}
& \text { LH kelkin } \\
& \text { quelguin (GP) }
\end{aligned}
$$$$
\begin{aligned}
& \text { 20. comprar } \\
& \text { *liwa- }
\end{aligned}
$$
LH liwa-
ligna-, liva- (GM) ligua (SI)
LS ts'aj
LS k'elkin
LS liwa-

La $n$ de la primera forma de Membreño es una mala interpretación de la $u$ que debe de haber tenido la intención de escribir. Esta confusión se da con cierta frecuencia. 
21. cortar

*tajk-

LH taj-

tai- (GM) tei (SI)

22. coyol

*juku

LH juku

yugu (GP)

23. coyote

*sua LH suaj

shu-guay (SI)

LS Jua

La secuencia gu recogida en Similatón puede representar la percepción de la parte final de alargamiento que facultativamente presentaban las vocales (una situación semejante parece darse en el lenca salvadoreño en casos como la palabra 'ratón' que Lehmann transcribió k'uua k'ua, Peccorini cúa y Campbell k'uwa). Por lo que respecta a la /j/ final, véase 2.3.14.
24. chupar
*ts'u
LH su-
LS ts'u- su- (GM, SI) zu- (OP)
25. decir *aj-
LH aj-
hay-(GP)
LS aj-
26. desear *saj
LH saj- shai-(GP, GM) shey (SI)
LS $\int e j-$
27. diente *nek

$$
\begin{aligned}
& \text { LH nek } \\
& \text { neeg }(G P) \text { neg (GM) }
\end{aligned}
$$
LS neh
28. dos *pe
LH pe pe (GP, SI) pee (GM)
LS pe
29. él *inani
LH inan ina
inán $(\mathrm{SI})$ iná $(\mathrm{GP}, \mathrm{GM})$
LS inani

30. enfermo, estar

*ona -LH un-

LS onaun-(GM) hun-(SI)

31. espina *ma

LH mana LS ma mano (GM) mana (SI): véase 1.1.6 Lenca hondureño -na'?'.

32. este *na

\section{LH na}

LS na-

naa (GM)

La raíz $n a$ - del LS se ha separado de la forma nanum 'aquí' (cf. inum 'allí').

33. estrella *sirik

LH siri

LS sirih siri (GM, SI), ciri (GP)

Véase la explicación dada en el rubro 12.

34. flor *sula

LH suna LS Jila
35. fuego *juk'a LH juka
yuga $(\mathrm{GP}, \mathrm{GM})$

LS ik' an

No se tiene explicación para la /n/ final de la forma del lenca salvadoreño.

36. frío, hacer

*ts'ana- LH sansan- (GM)

LS ts'ana-

37. grande *pukV

LH puki LS puka pugui-(GT) pugni (GM) pugue (SI) No se tiene explicación para la diferencia entre las vocales finales.

38. guacal *k'akma

LH kakma

LS k'ama cacma (GP)

LS peleh

39. hermano *pelek

$$
\begin{aligned}
& \text { LH pela } \\
& \text { pela }(\mathrm{GP}, \mathrm{GM})
\end{aligned}
$$
'hermana' mayor'

40. hígado *muts'u

LH musu LS muts'u

41. hormiga *its'its'i musu, mussu (GM)

LS its'its'i LH sisi
sisi (SI) sisí (GM) sizí (GP)

La aféresis de la $* / \mathrm{i} /$ inicial que se da en la forma del lenca hondureño, no se ha observado más que en este caso.

42. hueso

*ts'ek

LH sek
seig (GP) sej(SI)

LS ts'eh

La $i$ de la forma de Guajiquiro debe haber sido la percepción de la parte final del alargamiento que pueden presentar las vocales en el lenca hondureño. La forma $t s$ 'eh del lenca salvadoreño se ha separado de la expresión šíla-ts'ế que da Lehmann por mann por 'hueso' que en realidad significa 'hueso de muerto'. La / $\mathrm{h} /$ final aparece en otras formas compuestas con la raíz $t s$ 'eh como ints'eh 'pico' ('hueso de la boca').
43. ir *o- LH u- LS o-
44. jocote *muraka ?u- (CChD) u-(SI, GM) hu-(GP)
LH murak murag (GP) muraj (SI)
LS muraka

No se han observado otros casos de caída de $* /$ a/ final precedida por $* / \mathrm{k} /$, pero tampoco hay contraejemplos a la suposición de que se haya producido apócope en el lenca hondureño. 

45. lavar
*ts'ajk- LH sak-
sag- (GM, SI)
46. leña
LH sak
zag (GP) shag (GM) shaj (SI)
LS Jah
47. Iluvia
*so
LH so
sho (GP, GM)
48. macho
*kew
LH kew
kew $(\mathrm{CChD})$
LS Jo
LS kew

49. maíz

*ajma

LH ama

LS ima

ama (GP, GM, SI)

50. mapachín

*wala

LH wala

guala (SI) gwá · la (CChD)

LS wala

51. milpa

LH ta

taa (GM) ta (SI)

LS ta

52. montaña

*kotan

LH kotan $\operatorname{cotan}(\mathrm{GP})$ cotang (GM)

LS kotan

53. mover

*lum-

LH lum-

lum- (GM)

LS lum-

54. nariz

*nep

LH nepsek

LH -sek 'hueso'. LS -kuru '?

LS nepkuru

55. niño

*we

LH wewe

LS wewe guegue (GP) güegüe suiga 'niño de pecho' (GP). El lenca de Similatón presentaba la forma sin reduplicación we (escrita en las fuentes güe).

56. nosotros

*apinani

LH apinan

apinán (GP, GM, SI)

LS pinani

57. nube

\section{LH sopata} shopada (GP, GM)

LS ts'upa

El emparejamiento de $\mathrm{LH} / \mathrm{o} /$ con $\mathrm{LS} / \mathrm{u} /$ es irregular. La $o$ de la primera lengua podría representar una variante muy abierta de /u/. LH /-ta/ '?'.

58. oír

*eni- LH en-

hen-(GP) en-(SI, GM)

LS eni-

59. orinar

*wajsa-

LH wasa-

LS wila-

60. pavo

guasha-(GP) huasha-(GM)

LS lolo

LH lok

$\log (\mathrm{GM}, \mathrm{GP}) \operatorname{loj}$ (SI)
La forma esperada en el lenca salvadoreño - que ha formado el tema por reduplicación - sería /loloh/. En las fuentes con frecuecia se omite la /h/ en posición final de palabra.

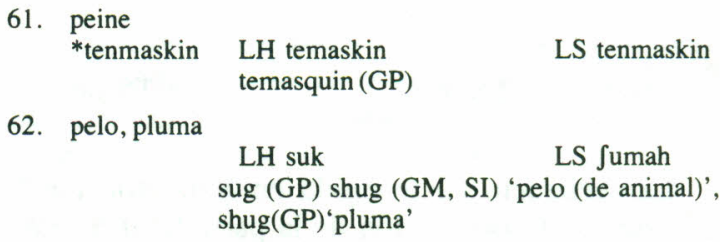

62. pelo, pluma

LH suk LS Jumah sug (GP) shug (GM, SI) 'pelo (de animal)', shug $(\mathrm{GP})$ 'pluma'

No hay una explicación satisfactoria para la presencia de /-mah/ en el lenca salvadoreño. Podría tratarse de un formativo de temas.

63. perro
$*$ su

LH suj

LS $\int u \int u$

shuy (GP) shui (GM) shuiy (SI)

Sobre / -j/en el lenca hondureño, véase 2.3.14

64. pico

$\begin{array}{lll}* \text { ints'ek } & \text { LH insek } & \text { LS ints'eh } \\ & \text { yn-seg (GP) }\end{array}$

65. piedra

*ke LH ke

LS ke

kee (GM) que (GP, SI)

66. piña

*mats'ati LH masati masate (GM)

LS mats'ati

La $e$ final de la forma recogida por Membreño debe ser resultado de la percepción de una variante abierta de /i/, que el oído del castellanohablante tiende a interpretar como $e$ en posición final inacentuada.

67. piojo

LH tem

LS tem

tem (GP, GM, SI)

68. puerco de monte

*map'it LH napir (GM)

*nap'it

LS map'it

La diferencia en las consonantes iniciales podría deberse a disimilación o asimilación por $* / \mathrm{p} \%$. La $r$ final del LH podría ser una mala percepción del alófono sonoro de / $\mathrm{t} /$ (véase 1.1.5.).

69. pulga

$$
\text { *t'ut'u LH tutu }
$$

LS t'ut'u

70. quebracho *sili

LH sili

LS sili 
71. quién

*k'ulan

LH kunan

cunan (GP, SI)

72. reír *jolo-

LH jolyol- (GM)

LS jolo-

73. río

*wara

LH wara guara (GP, GM, SI) wará- (CChD)

74. roble

*mal

LH mal

mal (GP, SI)

75. ropa

*lam-

LH lam-

LS lam-

lamín 'enagua' (GP, SI) lamtá

(GM) 'refajado'

LS: lamakin 'enagua', lamtára 'ropa'.

76. rostro *tik

LH tik

tig (GP)

LS tits'e

La forma del lenca salvadoreño debe de haber sido más bien / tits'eh/. / ts'eh/ 'hueso' aparece en otros compuestos como segundo miembro.

77. saber

*ti-

LH ti-

ti-(GM)

LS ti-

78. seis

*wi

LH wi

güilli (GP), huie (GS)

LS wi

La parte final de alargamiento facultativo de las vocales acentuadas del lenca hondureño (especialmente notable en los monosílabos, según se desprende de los datos) debe de haber sido interpretada como $e$.

79. sembrar

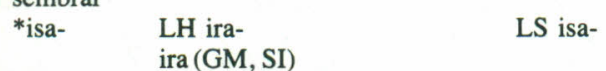

80. tapesco, cama
*le-
LH lema
LS leh
lema (GP, GM) ‘cama', lem (SI) 'tapesco'

No se tiene explicación para los segmentos-m(a) del lenca hondureño $\mathrm{y}-\mathrm{h}$ del lenca salvadoreño.

81. tigre (jaguar), león (puma)

*lepa LH lepa

lepa (GP, GM, SI, CChD)

LS lepa

82. tocar

*jete-

LH jet-

yet-(GM)

LS jete-

83. trabajar

LH tetted-(GM)
Como se ha señalado (Campbell, 1976b: 26), este término debe de ser un préstamo del pipil.

$\begin{array}{llll}\text { 84. } \begin{array}{l}\text { tres } \\ \text { *lawa }\end{array} & \begin{array}{l}\text { LH lawa } \\ \text { laagua (GP) lagua (GM, SI) }\end{array} & \text { LS lawa } \\ \text { 85. } \begin{array}{l}\text { tú } \\ \text { *amanani }\end{array} & \begin{array}{l}\text { LH amnan } \\ \text { amná(GP, GM) amnán (SI) }\end{array} & \text { LS manani } \\ \text { 86. } \begin{array}{l}\text { uña } \\ \text { *kumam }\end{array} & \begin{array}{l}\text { LH kuma } \\ \text { cuma (GP) }\end{array} & \text { LS kumam } \\ \text { 87. } \begin{array}{l}\text { venir } \\ \text { *po- }\end{array} & \begin{array}{l}\text { LH pu- } \\ \text { pu-(GM, SI) }\end{array} & \text { LS po- } \\ \text { 88. } \begin{array}{l}\text { yo } \\ \text { *unani }\end{array} & \begin{array}{l}\text { LH unan } \\ \text { uná(GP, GM) unán (SI) }\end{array} & \text { LS unani } \\ \text { 89. } \begin{array}{l}\text { zarigüeya } \\ \text { *ts'ewe }\end{array} & \begin{array}{l}\text { LH sewe } \\ \text { zegüe (GP) segue (GM) }\end{array} & \text { LS ts'ewe }\end{array}$

90. zopilote *kus

LH kus

cus (GP, GM, SI)

LS kuskus

En este caso, como en otros, las lenguas difieren por el funcionamiento en una de ellas de la reduplicación como formativo de temas.

4. Conclusiones sobre el sistema fonológico y el vocabulario reconstruidos

4.1. Tipología del sistema fonológico del protolenca y alteraciones principales sufridas en las lenguas descendientes

El sistema fonológico reconstruido tiene en particular por la oposición entre consonantes glotalizadas y no glotalizadas, y el tipo de cuadro vocálico mayor afinidad con los de las lenguas de las familias de la subárea mesoamericana denominada por Holt y Bright (1976:155) "mayense" que con las de las familias del área centroamericana de afinidades sudamericanas.

El cambio de mayor importancia que dicho sistema ha sufrido es, desde este punto de vista, la fusión incondicionada de las glotalizadas con las correspondientes no glotalizadas en lenca hondureño.

Los procesos de escisión de mayor trascendencia han sido dos de escisión secundaria que se dieron en el lenca salvadoreño: $* / \mathrm{s} /,>/ \mathrm{s} /, / 1 /, / \mathrm{\int} / \mathrm{y} * / \mathrm{k} /$ $>/ \mathrm{k} /, / \mathrm{h} /$. Estos llevaron a la aparición de los 
nuevos fonemas $/ \mathrm{S} / \mathrm{y} / \mathrm{h} /$ que aumentaron la complejidad del sistema de la lengua, que, como se ha señalado, no redujo las oposiciones existentes en la protolengua.

El lenca salvadoreño presenta un fonema más $/ \mathrm{t} \int$ '/, sobre cuyo origen no se puede señalar nada aquí por no haberse encontrado en lenca hondureño cognados de los rubros léxicos que lo contienen, que de todos modos son bastante escasos.

Los cambios aludidos aumentaron el aspecto "mayense" del lenca salvadoreño, en tanto redujeron el aspecto mesoamericano del lenca hondureño. En este último, la pérdida de las oposiciones por glotalización podría haberse debido a la influencia de las lenguas colindantes no mesoamericanas, como las misumalpas o el paya (chibcha).

\subsection{Rasgos del léxico reconstruido}

Los conjuntos de cognados, en su mayor parte, corresponden a rubros de vocabulario básico. Entre ellos hay 47 términos de la lista glotocronológica original de 215 .

Otra serie corresponde a nombres de vegetales y animales propios de la región, como coyol, quebracho, piña, ardilla, zarigüeya, coyote, jaguar, etc.

Además, existen unos pocos términos que dan indicios, muy limitados eso sí, sobre la cultura de los hablantes del protolenca. Tal es el caso de sembrar, maíz, milpa y comal, a partir de los cuales se infiere que eran agricultores, que cultivaban el maíz y que, probablemente, este cereal era su cultivo más importante, pues */ta/ que parece afín al término que en las lenguas chibchas designa el campo de labranza en general (bribri $/ \mathrm{t} \overline{\mathrm{i}} /$, muisca $/$ ta/, p.e.), en caso de haber alguna relación, se habría especializado para 'milpa'. Comprar nos indica que practicaban el comercio y caites, ropa, peine y comal nos dan una idea sobre el tipo de objetos que fabricaban.

\section{BIBLIOGRAFIA}

Andrews V. y E. Wyllys. 1970. "Correspondencias fonológicas entre el Lenca y una lengua Mayance". Estudios de Cultura Maya 8: 341-387. Méjico: Universidad Autónoma de Méjico.

Campbell, Lyle. 1976a. "The last Lenca". International Journal of American Linguistics. 42(1): 73-78. 1976b. "The linguistic Prehistory of the Southern Mesoamerican Periphery" Fronteras de Mesoamérica, $14^{\mathrm{a}}$ Mesa Redonda, 1:157-184. Méjico: Sociedad Mexicana de Antropología.

1979. "Middle American Languages". En: The Languages of Native America: Historical and Comparative Assessment. Compilado por Lyle Campbell y Marianne Mithun: 902-1000. Austin: University of Texas Press.

Campbell, Lyle et alii. 1978. "Honduran Lencan". International Journal of American Linguistics. 44(4): 330-332.

Chapman, Anne. 1978. Los lencas de Honduras en el siglo XVI. Tegucigalpa: Instituto Hondureño de Antropología e Historia.

Delbrück, B. 1882. Introduction to the Study of Language. Breitkopf y Hartel: Leipzig.

Del Río Urrutia, María Ximena. 1985. El lenca de Chilanga: fonología y léxico. Tesis de licenciatura. Universidad de Costa Rica.

Escalante, R. y L. Faier. 1959. "Relaciones del Lenca y Xinca". Ponencia presentada en la reunión anual de la American Anthropological Association.

Greenberg, J.H. 1960. "The General Classification of Central and South American Languages". En: Men and cultures: Selected Papers of the $5^{\text {th }}$ International Congress of Anthropological and Ethnological Sciences, Philadelphia, September 1956. Compilado por Anthony F.C. Wallace: 791-794. Filadelfia: University of Pennsylvania Press.

Hoenigswald, Henry M. 1950. "The Principal Step in Comparative Grammar". Language. 26: 357 . 364.

1960. Language Change and Linguistic Reconstruction. Chicago: University of Chicago Press.

1973. Studies in Formal Historical Linguistics. Dordrecht, Holanda: D. Reidel Publishing Company. 
Holt, Dennis y William Bright. 1976. "La lengua paya y las fronteras lingüísticas de mesoamérica". Las fronteras de Mesoamérica. $14^{a}$ Mesa Redonda, Sociedad Mexicana de Antropología 1: 149-156. Méjico.

International Phonetic Association. 1949. The Principles of the International Phonetic Association. Londres: Department of Phonetics, Univesity, College.

Jijón y Caamaño, Jacinto. 1943. El Ecuador Interandino y Occidental. Vol. 3: Las lenguas del sur de Centroamérica y el norte y centro del Oeste de Sud-América. Quito: Editorial Ecuatoriana.

Johnson, Frederick. 1940. "The linguistic Map of Mexico and Central America”. En: The Maya and Their Neighbors. Publicado por C.L. Hay: 88-114, New York: Appleton Century Company, Inc.

Kaufman, Terrence. 1974. Idiomas de Mesoamérica. Editorial "José Pineda Ibarra". Ministerio de Educación: Guatemala.

Lehmann, Walter. 1920. Zentral-Amerika, Parte I, Die Sprachen Zentral-Amerikas. Vol. 2. Berlin: Verlag Dietrich Reimer.

Loukotka, Čestmir. 1948. "Sur la classification des langues indigènes de l'Amérique du Sud". Actas del XXXVIII Congreso Internacional de Americanistas: 193-199.

Mason, John A. 1939. "Los cuatro grandes filones lingüísticos de México y Centroamérica". Actas del XXVII Congreso Internacional de Americanistas: 282-288.

1940. "The Native Languages of Middle America". En: The Maya and Their Neighbours. Publicado por C.L. Hay: 52-87. New York: Appleton Century Company, Inc.

1943. "Idiomas indígenas y su estudio”. América Indígena 3(3): 231-244.

McQuown, Norman A. 1955. "The Indigenous Languages of Latin America". American Anthro-

pologist 57: 501-570.
Meillet, Antoine. 1921. Linguistique historique et linguistique générale, Vol. 1. París: Champion.

1925. La méthode comparative en linguistique historique. Oslo: Instituttet for Sammenlignende Kultur-forskning.

Membreño, Alberto. 1897. Hondureñismos: Vocabulario de los provincialismos de Honduras. Tegucigalpa: Tipografía Nacional.

Pinart, Alphonse L. y Hernández E. 1897. Pequeño vocabulario de la lengua Lenca. Petite Bibliothéque Américaine VIII. París.

Schmidt, Wilhelm. 1926. Die Sprachfamilien und Sprachenkreise der Erde. Heidelberg: Winter.

Squier, Ephraim G. 1858. The States of Central America. Nueva York: Londres.

Swadesh, Morris. 1958. "La lingüística de las regiones entre las civilizaciones mesoamericanas". Actas del XXXIII Congreso Internacional de Americanistas.

1959. Mapas de la clasificación lingüística de México y de las Américas. Méjico, D.F.: Universidad Nacional Autónoma de Méjico.

1967. "Lexicostatistic Classification". Handbook of Middle American Indians. Vol. 5: Linguistics. Compilado por Norman McQuown: 79-116. Austin: University of Texas Press.

Tax, Sol. 1960. "Aboriginal Languages of Latin America”. Current Anthropology 1(5-6): 431436.

Thomas, Cyrus. 1902. "Provisional List of Linguistic Families, Languages, and Dialects of Mexico and Central America"/ American Anthropologist, N.S. 4: 207-216.

Voegelin, C.F. y F.M. Voegelin. 1965. "Languages of the world: native America, fascicle 2." Anthropological linguistics 7(7). 


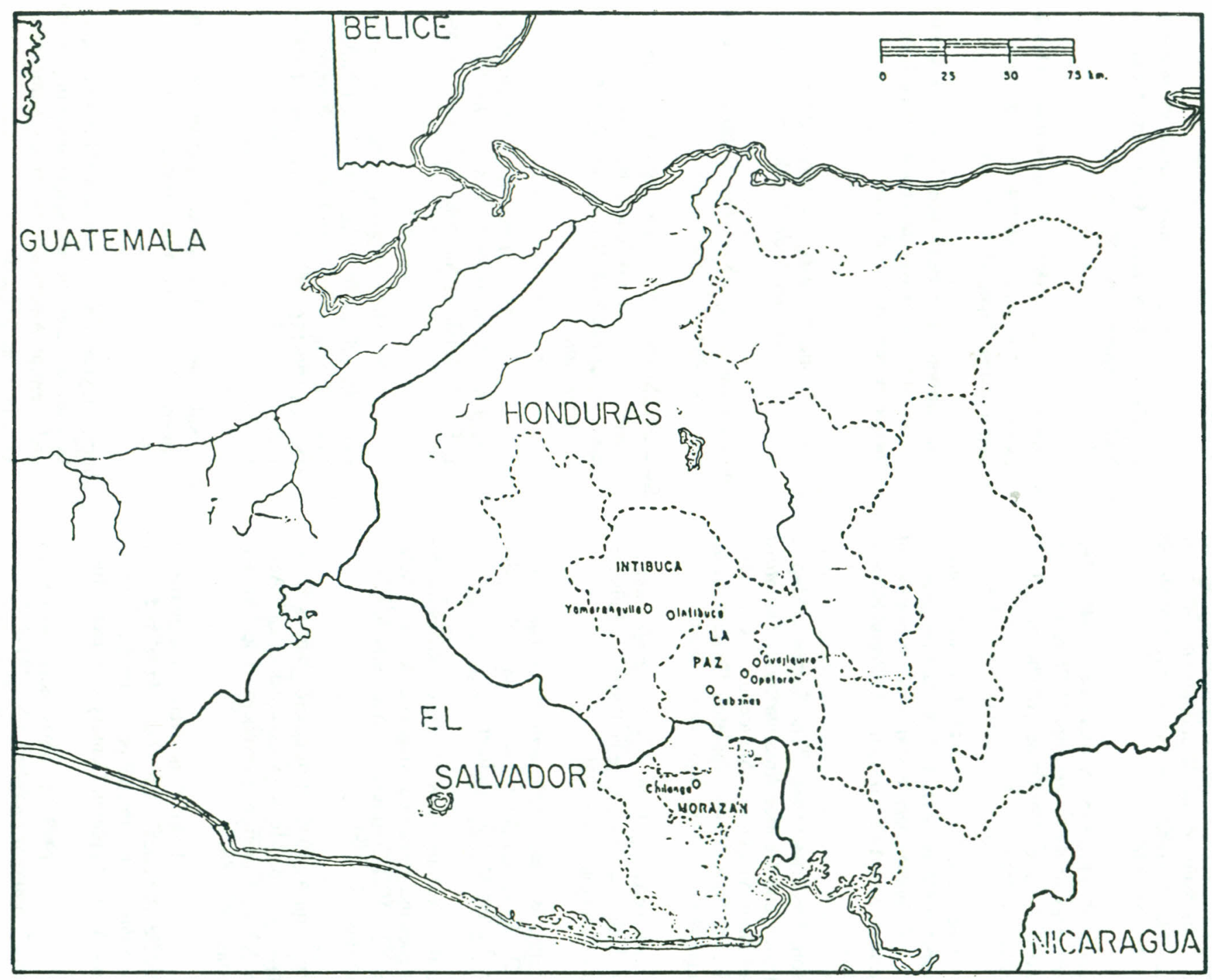




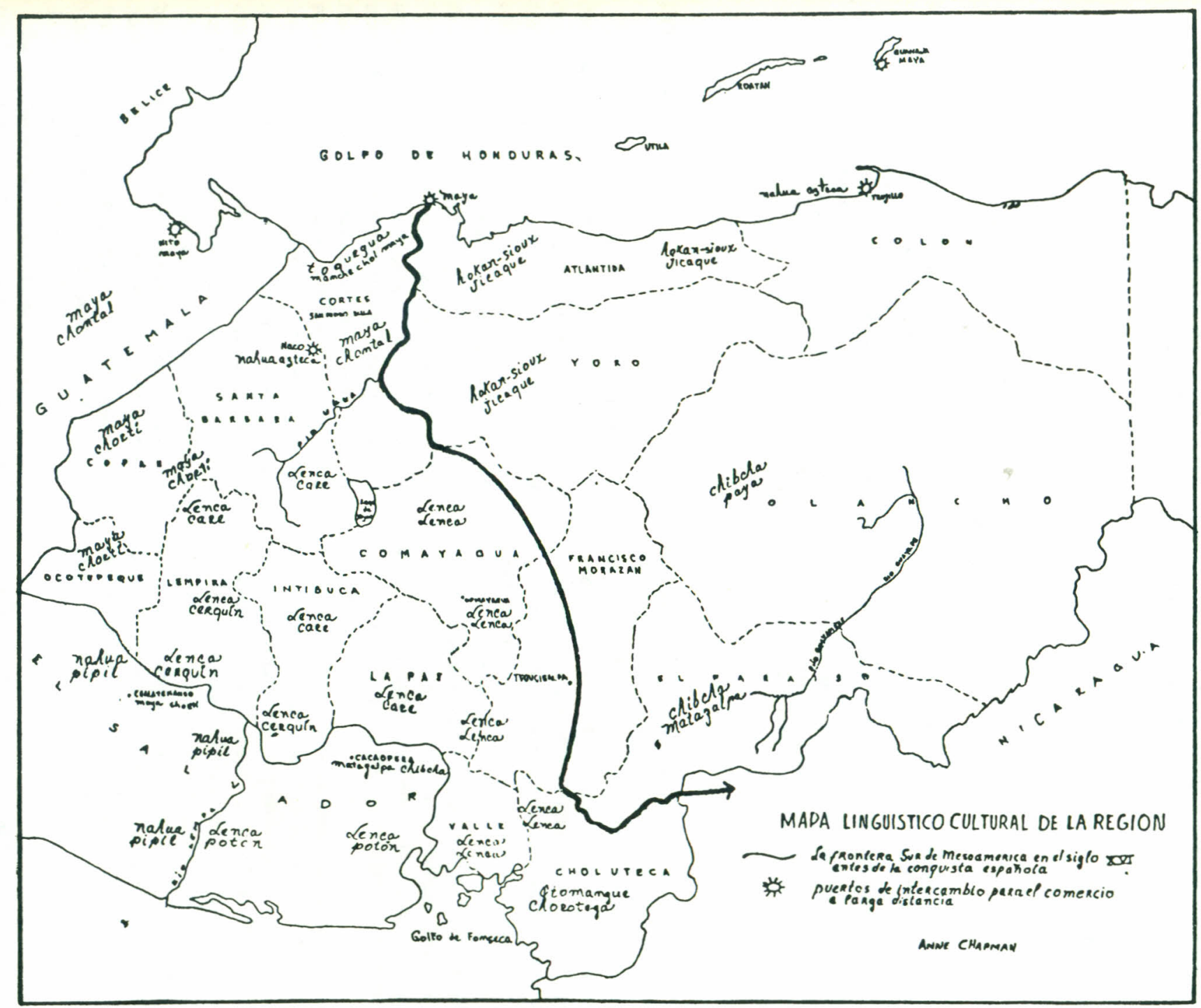


